\title{
Minimum Viable Product and the Importance of Experimentation in Technology Startups Dobrila Rancic Moogk
}

\author{
"Startups don't starve; they drown." \\ Shawn Carolan \\ Managing Director, Menlo Ventures
}

\begin{abstract}
Entrepreneurs are often faced with limited resources in their quest to commercialize new technology. This article presents the model of a lean startup, which can be applied to an organization regardless of its size or environment. It also emphasizes the conditions of extreme uncertainty under which the commercialization of new technology is carried out. The lean startup philosophy advocates efficient use of resources by introducing a minimum viable product to the market as soon as possible in order to test its value and the entrepreneur's growth projections. This testing is done by running experiments that examine the metrics relevant to three distinct types of the growth. These experiments bring about accelerated learning to help reduce the uncertainty that accompanies commercialization projects, thereby bringing the resulting new technology to market faster.
\end{abstract}

\section{Introduction}

Commercializing a brand new product or service is a complex task with an uncertain outcome. Whether it is undertaken in a big corporation, a startup, or a not-forprofit organization, it requires vision, determination, and resources. Entrepreneurs of all stripes, in a garage, a multinational, or in a social enterprise, have brilliant ideas and doggedness to succeed. Resources, however, can be scarce.

Money needed to bring a new idea to market is difficult to secure. In an established business, metrics of success that apply to the mainstream products or services typically do little to justify an investment in a new idea serving different customers in different markets. For startups, with the ever-diminishing availability of venture capital over the last decade, bootstrapping is the order of the day. Those few that have been lucky to secure venture capital investment must account for frugal-yet-effective spending to their boards of directors. For these reasons, all visionaries who want to succeed need to ensure that the scarce resources not only last longer but also bring a demonstration of market viability as early as possible.
A response to these challenges is presented by Eric Ries, in his book The Lean Startup (tinyurl.com/7dxddzz), which has its origins in his blog (startuplessonslearned.com), and his concrete entrepreneurial experiences in startups such as IMVU (imvu.com), which he co-founded and where he served as a CTO. Much of Ries's original thinking draws inspiration from Clayton Christensen's theories presented in The Innovator's Dilemma (tinyurl.com/7onvohk) and The Innovator's Solution (tinyurl.com/7n7x5rd); Geoffrey Moore's Crossing the Chasm (tinyurl.com/6qfeowt); and the lean production systems derived by Taiichi Ohno and Shigeo Shingo and popularized by Toyota (tinyurl.com/3a8tx3). This article will focus on the lean startup and the novel idea of "minimum viable product"; the article will show how these concepts can make technology entrepreneurs and their startup endeavours more successful.

\section{A Lean Startup}

For the purposes of this article, Ries's definition of a startup will be used: "A startup is a human institution designed to create a new product or service under conditions of extreme uncertainty" (Ries, 2011; tinyurl.com/ $7 \mathrm{dxddzz}$ ). This goes hand in hand with the intention to re- 


\section{Minimum Viable Product and the Importance of Experimentation}

\section{Dobrila Rancic Moogk}

cognize that startups, apart from what is commonly considered to be a bootstrapped, or a venture capital backed small operation, can be found in for-profit organizations of all sizes, among the not-for-profits, and even within the government. This wide definition of startups should be kept in mind even though the examples in this article are predominantly drawn from new technology companies.

Typically, startups arise around a vision that a new product or service (henceforth, a product) will be embraced by a particular market because it solves the customers' urgent problem. Following the path of established companies, startups often devise a strategy to develop a full product based on this vision and deploy it in the target market. In many such cases, there is not as much traction as anticipated, which brings about the painful realization that either the product was not compelling, the market was poorly chosen, or both.

Developing a full product before testing a concept in the market is a risky proposition due to the extreme uncertainty associated with startup operations. But how can the market be tested if the product is not fully designed and implemented? This apparent paradox is based on the notion that startups operate on the same management principles as the established companies. Although established companies typically serve known customers in deterministic markets, startups have to address an environment of extreme uncertainty. Hence, startups need to operate in a way that will provide them with the opportunity to learn while validating their vision; ambiguity must be replaced by increasing certainty over as short a timeframe as possible.

The best learning for a startup comes as a result of experiments that test a version of a product against relevant metrics. The result of the experiment can reveal whether the original idea: i) is valid, in which case development can continue in the same direction or ii) is not valid, in which case the strategy has to change. The acceleration of this feedback loop is essential to take full advantage of the learning.

The key principles of the lean startup include: omnipresence of the entrepreneurs, uniqueness of the startups management style, and learning from product testing against relevant metrics. This article will drill down further into the aspect of accelerated learning from experiments designed to validate a product against pertinent metrics.

\section{A Minimum Viable Product}

A startup operates around a vision that its product will uniquely solve the pressing problems of customers in their target market. The founders often expect that their product will deliver an unprecedented return on their investment. This vision includes two important assumptions: the assumption around providing value (i.e., the value hypothesis) and the assumption around growth in the market (i.e., the growth hypothesis).

To illustrate these two assumptions, consider a now well-known example of the early success Facebook had with investors. In the summer of 2004, when Facebook was just six months old, had 150,000 registered users, and had very little revenue, the company was able to secure its first $\$ 500,000$ investment (Ries, 2011; tinyurl.com/7dxddzz). In April 2005, Facebook raised an additional \$12.2 million (Arrington, 2005; tinyurl.com/ yduzltb). Apart from the business model that was based on producing revenue from different types of ads and sponsored groups, what was it that investors found so compelling about the company? Remarkable as it was that, in such a short time, Facebook amassed 3.85 million users, equally impressive was the statistic that $60 \%$ of the users logged on daily (Arrington, 2005). Moreover, Facebook did not spend money to acquire its customers. The organic growth in registered users coupled with their strong engagement validated the company's value hypothesis. The increase from 150,000 users at 6 months to 3.85 million users at 14 months validated Facebook's growth hypothesis.

For a startup, it is essential to validate its value and growth hypotheses as soon as possible. In order to do that, the company has to come up with a version of its product that is complete enough to demonstrate the value it brings to the users: a minimum viable product (MVP). It then needs to design experiments that will use the MVP to confirm (or refute) its value and growth hypotheses. On the one hand, an MVP may need less time to develop and should have just the "bare bones" set of features. On the other hand, an MVP should include development of capabilities to measure its traction in the market. Although many product features that were "on the drawing board" will be soon requested by the users, designers should avoid the temptation of including these features in the initial development - their time will be much better spent developing the experiments that measure the MVP's impact. 


\section{Minimum Viable Product and the Importance of Experimentation}

\section{Dobrila Rancic Moogk}

In order to measure the effect of the MVP, baseline data must be captured. Further data will be collected as part of planned tests on the initial MVP and its subsequent revisions. The complexity and number of tests can be staggering. In an online consumer business, the number of different versions of a product running different tests can be in the thousands, and can change every week, even daily.

Regardless of the challenge of managing that complexity, the more pressing question is how to determine what needs to be measured in order to evaluate the impact of these tests. To address this issue, Ries suggests that three types of engines of growth should be considered: sticky, viral, and paid (Ries, 2011; tinyurl.com/ 7 dxddzz).

The sticky engine of growth relies predominantly on the high retention rate of its customers. A mobile telephone service provider would be an example of this type of growth. Another example would be a fabless semiconductor company that sells intellectual property (such as ARM and its microprocessors) to be designed into another product (such as a smartphone). The company relying on the sticky growth needs to diligently track the number of customers who disengage from the company's product. If that number is related to a period of time and is expressed as a fraction of all the customers, it is called the churn rate. The company's growth rate is defined by the growth rate of newly acquired customers minus the churn rate. In contexts that depend on a sticky engine of growth, this is a relevant metric that should be captured in MVP experiments.

The viral engine of growth was originally described as "network-enhanced word of mouth" by Draper Fisher Jurvetson (Jurvetson, 2000; tinyurl.com/6nneasa), a venture capital firm that was a seed investor in Hotmail.com. It is now a legendary story that the growth of Hotmail.com accelerated when the company decided to add a link at the bottom of every outgoing message sent by existing users, inviting the recipients to register for its free email service. Hotmail.com went from zero to 12 million users in 18 months with a $\$ 50,000$ advertising budget (Jurvetson, 2000). Other examples of this growth are online social networks and "house parties" used to sell a slew of different products.

The paid engine of growth simply relies on the difference in the lifetime revenue from each customer minus the cost of acquiring every additional customer. Clearly, the higher the difference, the higher the rate of growth; however, the cost of acquiring an additional customer should include all related costs, including such things as Google AdWords, supporting a sales force, and efforts to bring customers into a physical store. The MVP experiments of companies that rely on the paid engine of growth should track metrics related to lifetime revenue values and customer acquisition costs.

It is important to emphasize that metrics designed to evaluate the impact of MVPs should measure the real business impact and not simply produce feel-good results through "vanity metrics". An example of the latter case would be a company that relies on the sticky engine of growth tracking only the number of newly acquired customers. While steady growth in this metric is encouraging, the company may not be making any real progress if the number of disengaged customers is growing at the same rate.

\section{Conclusion}

Startups are organizations that develop new products under conditions of extreme uncertainty. The startup label can be applied to small new companies, but it can also be applied to parts of established enterprises that are trying to break new ground in order to give a boost to a slowing growth. In every case, startups can benefit from the lean startup philosophy, especially from the ideas and learning generated as a result of testing minimum viable product versions against relevant metrics. By applying this philosophy, startups can develop products that are tailored to target markets.

The idea of a lean startup focuses on increasing development efficiencies and reaching the target market sooner, thus potentially capitalizing on the first-mover advantage. It emphasizes that startups should try to eliminate waste (e.g., wasted development resources) by releasing an MVP as soon as possible. Startups should use MVPs to engage target customers and test the value and growth hypotheses using metrics that are suited to the type of the engine driving a startup's growth (i.e., sticky, viral, or paid). Initially, development should focus on experiments that provide answers to fundamental questions related to the value and growth hypotheses. Subsequently, the focus should be maintained on the engine of growth itself. Although it is possible that more than one type of engine can be propelling the growth, the most successful startups are those that focus on only one of them at a time. 


\section{Minimum Viable Product and the Importance of Experimentation}

\section{Dobrila Rancic Moogk}

The fundamental idea behind the lean startup philosophy is that the real product of an early-stage startup is an experiment, or a slew of experiments, that contribute to reducing the initial extreme uncertainty. Progress of a startup can be measured by the learning that is gained from these experiments. The more accelerated the learning, the closer the startup gets to releasing the right product in the right market and to attaining its visionary goals.

\section{About the Author}

Dobrila Rancic Moogk has over 19 years of executive and leadership experience in $R \& D$, marketing, product management, and people management roles in high-tech companies ranging from startups to multinationals in North America and Europe. She is currently working on business strategy, product development, and corporate development with several high-tech startups. Her interests are in the area of increasing the efficiency of innovation commercialization. Also, Dobrila serves as a vice-chair with the University of Ottawa Women in Engineering and Computer Science committee and a vice-chair on the Volunteer Ottawa Board of Directors. Dobrila has a BSEE as well as Master of Engineering and MBA degrees from the University of Ottawa.

Citation: Rancic Moogk, D. 2012. Minimum Viable Product and the Importance of Experimentation in (cc) BY 